\title{
BUZÓN GARCÍA, J. M., GÓMEZ DEVÍS, M. B. Y GÓMEZ MOLINA, J. R. (2017): ACTITUDES LINGÜÍSTICAS EN VALENCIA Y SU ÁREA METROPOLITANA. ESTUDIO LONGITUDINAL Y ANÁLISIS DE TENDENCIAS. VALENCIA: TIRANT HUMANIDADES. 309 PÁGINAS
}

\author{
FRANCISCO GIMENO \\ JOAQUÍN RODRIGO \\ Universidad de Alicante \\ fgimeno@ua.es \\ joaquin.rodrigo@ua.es
}

El libro plantea la complementariedad entre lingüística, sociología, antropología y psicología, y dicha interdisciplinariedad ha supuesto una gran contribución de los estudios sociolingüísticos a la investigación lingüística hispánica. Los autores son excelentes profesores del Departamento de Filología española de la Universidad de Valencia que han centrado sus estudios en temas de sociolingüística hispánica y de lingüística aplicada.

La monografía consta de los siguientes apartados: 1) índice general (pp. 9-10), 2) prólogo de A. López García Molins (pp. 11-2), 3) introducción (pp. 13-45), 4) marco epistemológico (pp. 47-74), 5) planteamiento metodológico (pp. 75-105), 6) análisis estadísticos e interpretación de resultados (pp. 107-235), 7) conclusiones finales (pp. 237-69), 8) referencias bibliográficas (pp. 271-82), y 9) anejos (pp. 285-309).

La investigación actual sobre las actitudes lingüísticas y su importancia para la sociolingüística del multilingüismo social han sido indiscutibles, con el fin de analizar la situación sociolingüística de la Comunidad Valenciana, desde las primeras aportaciones de los años sesenta del pasado siglo de L. V. Aracil y R. L. Ninyoles.

La nomalización lingüística fue llevada a cabo por la Generalitat Valenciana, a partir de la Llei d'us i Ensenyament del Valencià (1983), en la que se proponían medidas para impulsar el uso del valenciano en todos los ámbitos de uso, así como la incorporación obligatoria del valenciano en el sistema educativo de la Comunidad Valenciana (excepto en las comarcas tradicionalmente castellanohablantes), y ha resultado poco eficaz, ya que han encontrado resistencia y tensiones en un discurso de confrontación política y en su pérdida de base social. El aumento del conocimiento escolar del valenciano no ha servido para mantener la vitalidad de la lengua, ya que se ha comprobado un retroceso y una reducción progresiva de

Para citar esta reseña / To cite this book review: Gimeno, Francisco y Joaquín Rodrigo (2018). Reseña de Buzón García, J. M., Gómez Devís, M. B. y Gómez Molina, J. R. Actitudes lingüísticas en Valencia y su área metropolitana. Estudio longitudinal y análisis de tendencias. Valencia, Tirant Humanidades, 2017. ELUA, 32: 375-379. doi: 10.14198/ELUA2018.32.18

Enlace / Link: http://dx.doi.org/10.14198/ELUA2018.32.18 
los usos sociales y de la trasmisión familiar. La hipótesis general de la monografía ha sido que la explicación de la variación en el conocimiento y uso del valenciano podían ser dos: 1) los cambios demográficos en la composición de la población urbana, a finales del siglo pasado y principios del XXI, y 2) la política lingüística llevada a cabo por la Generalitat Valenciana, durante el gobierno del Partido Popular.

La situación sociolingüística del área metropolitana de Valencia es un conflicto lingüístico, en el que se ha activado la configuración estática de la diglosia amplia, sin movilidad social, dentro de una dinámica progresiva de sustitución lingüística del valenciano por el castellano. Las hipótesis específicas de trabajo han sido: 1) las variedades estándares (valenciano y castellano) serán las más valoradas en la dimensión cognoscitiva: competencia, prestigio profesional, clase social y estatus, sin diferencias cuantitativas significativas entre ellas. Por su parte, las variedades no estándares (valenciano y castellano) serán las más valoradas en la dimensión afectiva: atracción personal y social, solidaridad, grado de identificación, lealtad y orgullo lingüísticos, con preferencia hacia el valenciano; 2) los índices actuales de percepción positiva del valenciano frente al castellano habrán disminuido sustancialmente en determinados aspectos instrumentales, prestigio lingüístico y valor integrativo, respecto de los observados dos décadas atrás; 3) el proceso de normalización lingüística no solo no avanza, sino que la sustitución del valenciano por el castellano se va incrementando progresivamente, a consecuencia tanto de una planificación lingüística aplicada en los últimos veinte años, como de la escasa implicación subjetiva y presión social en el uso del valenciano, y 4) los factores sociales con mayor peso probabilístico en las actitudes lingüísticas manifestadas serán, en orden decreciente: la lengua habitual, el nivel sociocultural, el modo de vida, la edad (cambio de actitud generacional) y el sexo.

El tamaño de la muestra ha sido de 108 jueces para los textos leídos y 108 para los textos orales espontáneos, de acuerdo con la siguiente distribución por variables sociales: 54 hombres y 54 mujeres, 54 castellanohablantes y 54 bilingües, así como 12 hablantes por cada estrato generacional (20-34 años, 35-55 años y más de 55 años). En la estratificación de ambos conjuntos se tuvieron en cuenta el sexo, la edad, el nivel sociocultural, la lengua materna, la lengua habitual, el modo de vida y la residencia. El nivel sociocultural se ha establecido a partir de la combinación de otros dos parámetros básicos (grado de instrucción y ocupación profesional), y se han establecido tres estratos (alto, medio y bajo). Las variables lengua materna y lengua habitual han proporcionado información, tanto sobre la competencia comunicativa como sobre el proceso de aculturación de las dos lenguas en contacto. Se han considerado tres variantes para la lengua materna (castellano, valenciano y ambas), y tres variantes para la lengua habitual: castellanohablante (bilingüe pasivo), valenciano (bilingüe con uso predominante del valenciano) y bilingüe (uso equilibrado de ambas lenguas). El modo de vida es un factor social delimitado por el modo de producción, y la forma en que un individuo ha organizado su vida. Se han establecido tres variantes: familia, ocio y trabajo. La variable demográfica de la residencia ha indicado si existen diferencias cuantitativas y cualitativas entre la ciudad y las comarcas colindantes, y se ha tabulado de dos formas: 1) Valencia ciudad y cada comarca (Horta Nord, Horta Oest y Horta Sud), y 2) Valencia ciudad y el conjunto de municipios del área metropolitana. Dicha estratificación coincide con las especificadas en el "Proyecto para el estudio sociolingüístico del español de Valencia y su área metropolitana". 
Los procedimientos empleados han demostrado ser válidos y fiables, no solo con fines descriptivos, sino fundamentalmente para la obtención de inferencia estadística, y se ha incorporado toda la información al paquete estadístico SPSS para Windows (vid. J. Rodrigo, 2015). La metodología cuantitativa e informática ha sido totalmente necesaria e imprescindible para la investigación sociolingüística de la variación y el cambio lingüístico. Las conclusiones finales más relevantes han sido las siguientes:

1) la categorización social obtenida en los distintos componentes y ordenada según puntuaciones decrecientes de los estímulos ha mostrado que los grupos perciben de manera diferente las variedades, según su grado de estandarización: en la dimensión cognoscitiva las variedades estándares han obtenido mejor puntuación, con predominio del castellano estándar, mientras que en la dimensión afectiva han sido las variedades no estándares, con predominio del valenciano no estándar. Por otra parte, la variedad castellana no estándar ha podido considerarse como una variedad estereotipada, ya que ocupa el último lugar en las distintas escalas, mientras que el vernáculo valenciano ha destacado por su carácter identitario;

2) Los resultados de la variedad lingüística más adecuada en los distintos ámbitos de uso han mostrado distinta distribución funcional de las dos lenguas en contacto, y no puede considerarse bilingüismo diglósico, sino de adecuación situacional del registro. Además, la variedad valenciana estándar también se ha evaluado adecuada para situaciones formales, de manera que la conservación de dicha variedad puede quedar garantizada. La mejor adecuación para las situaciones de uso formal ha correspondido a las variedades estándares con valor instrumental, pero con cierta mejora del castellano estándar ante el estímulo oral, mientras que las variedades no estándares se han adecuado mejor a las situaciones informales con valor integrativo y carácter relacional, pero con mayor predomino del valenciano no estándar ante el estímulo oral;

3) las percepciones sobre el estatus y la solidaridad de las variedades usadas en el área metropolitana de Valencia han cambiado entre 1995 y 2015. En la dimensión cognoscitiva, los atributos de estatus y puestos de responsabilidad profesional han sido ahora de mayor intensidad en el castellano estándar que en el valenciano estándar, y puede interpretarse como un avance en el prestigio social y función instrumental del castellano estándar sobre el valenciano estándar. Por consiguiente, el castellano estándar va desplazando paulatinamente al valenciano estándar. Por otra parte, las variedades no estándares han sido las menos valoradas, y el castellano no estándar ocupa la última posición como variedad estereotipada. En la dimensión afectiva, ha mejorado la evaluación del valenciano no estándar, tanto en atractivo personal y social como en la identificación con amigos, y dichos valores pueden garantizar su conservación. Mientras que los informantes de 1995 identificaban de manera prioritaria al locutor de valenciano estándar como su variedad propia, ahora lo ha hecho la voz del castellano estándar, y también ha mejorado su posición en la identificación con un amigo. Por otra parte, la variedad de castellano no estándar se ha mantenido como la menos identificada con el hablante y amigo, mientras que la variedad de valenciano no estándar ha mejorado su evaluación en tiempo real, tanto en atractivo personal y social como en identificación con amigos, y pueden contribuir a su conservación en la interacción cotidiana; 
4) en la función instrumental del valenciano, la respuesta afirmativa sobre la promoción o ascenso social que supone hablar en valenciano ha representado el $44 \%$, pero si observamos la evolución del período temporal se ha descubierto una leve disminución en la valoración positiva, y un incremento sustancial de la percepción negativa, lo que ha confirmado cierto decaimiento en dicha función. En cuanto a la incidencia de los estratos sociales, en la valoración negativa han destacado los castellanohablantes $(62,1 \%)$, el sociolecto alto y los jóvenes, y puede reinterpretarse como un componente dinámico regresivo en la función sociológica de la lengua. En conclusión, han sido, pues, varios los estratos sociales que han reducido su percepción de que el hablar valenciano supone un ascenso social;

5) en el grado actual de lealtad lingüística y la relación identitaria lengua-territorio, se ha obtenido un índice negativo de lealtad (56,5\%), y el estudio generacional nos ha mostrado que ha disminuido la adhesión al valenciano como símbolo de identidad social. En conjunto, gran parte de la población encuestada, especialmente los grupos de mujeres, los adultos, el nivel sociocultural bajo y los castellanohablantes han mostrado un elevado grado de asimilación lingüística y cultural al castellano, y han manifestado que no es necesario hablar valenciano para sentirse valenciano;

6) los valores de lealtad lingüística han funcionado combinados con el prestigio, el orgullo y la utilidad hacia el valenciano, y han confirmado la vigencia del conflicto lingüístico, con un dinamismo regresivo del valenciano y un dominio progresivo del castellano, a consecuencia tanto de la política lingüística desarrollada por el Gobierno Valenciano en los últimos veinte años, como de la escasa presión social por la conservación del valenciano;

7) sobre el grado de vitalidad futura del valenciano, la respuesta actual ha sido manifiestamente negativa (62\%), y el estudio generacional ha mostrado que todos los estratos sociales (excepto los bilingües y los adultos) indican un uso predominante del castellano en el futuro, poco favorecedora hacia la revitalización del valenciano. Dada la relevancia del factor edad, debe señalarse que los jóvenes son poseedores de una buena competencia comunicativa del valenciano, pero favorecen el incremento progresivo del castellano como lengua de uso, y confirman la reducción progresiva de los usos sociales del valenciano y de la trasmisión familiar;

8) el nivel activo de tolerancia hacia el valenciano ha reflejado una actitud cultural que puede favorecer la conservación de esta lengua (75\%), pero es mayor hacia el castellano $(93,1 \%)$, la cual contribuye al predominio del castellano en la interacción cotidiana. La mayor intolerancia hacia el valenciano ha procedido, en orden decreciente, de los castellanohablantes, del sociolecto bajo, de los hombres y de los residentes en la ciudad de Valencia. Sin embargo, el grado de intolerancia hacia la recepción de mensajes en valenciano ha sido mínimo $(5,1 \%), \mathrm{y}$

9) en conjunto, en la selección de la variedad del castellano estándar han predominado los elementos de la dimensión cognoscitiva sobre la afectiva, y tiene garantizado su predominio sociolingüístico. En la selección de la variedad del valenciano estándar han predominado los elementos de la dimensión afectiva sobre la cognoscitiva, y debiera potenciarse la identidad cultural como factor determinante en la actitud hacia la variedad propia, dentro de una planificación futura del valenciano, en el que los factores sociales que mejor podrían favorecerlo son el estrato sociocultural alto, 
las mujeres y los jóvenes. En la selección de la variedad del valenciano no estándar han predominado tanto los elementos de la dimensión afectiva, como los de la cognoscitiva, y debiera potenciarse un uso más habitual en la calle y en la familia, especialmente por las mujeres.

Sin embargo, los análisis psicosociales de la conciencia lingüística y actitudes lingüísticas deben determinarse en nuestros días por las coordenadas generales (antropológicas y sociológicas), y en particular por las nuevas tecnologías de la información y la comunicación, que favorecen la globalización económica y la comunicación humana. Los medios de comunicación social juegan un papel clave, en el proceso de formación y modificación de la opinión pública. Una de las principales características de la globalización ha radicado en el hecho de que ha favorecido un horizonte supranacional, donde la lengua franca a finales del siglo pasado y principios del siglo XXI ha sido el inglés estadounidense, aunque hoy sea un residuo del pasado inmediato. El futuro próximo abre nuevas posibilidades al desplazamiento de ese monopolio del inglés por el oligopolio de varias lenguas supranacionales (chino mandarín, hindi-urdu, inglés, árabe, ruso y español), y cierra posibilidades a la conservación de las lenguas minoritarias (vid. F. Gimeno, 2008; F. Gimeno y L. Valozic, 2012).

Naturalmente, es un levísimo retoque de una investigación, que por muchas razones aparece como monografía sólida y densa de profundización en los presupuestos sociolingüísticos. La contribución que nos han brindado los autores J. M. Buzón, M. B. Gómez y J. R. Gómez ha sido extraordinaria, y sintetiza un trabajo y preocupación de varios años. Sin duda alguna, los apartados más necesarios y destacables han sido el capítulo tercero sobre la metodología de la encuesta en el área metropolitana de Valencia, y el capítulo cuarto sobre el tratamiento estadístico de los datos, así como las muy amplias conclusiones finales, ya que en nuestros días la investigación sociolingüística debe ser objeto de observación y experimentación, y la formulación de hipótesis de trabajo indica las pautas a seguir. En nuestros días, todas las ciencias son cuantitativas, y sin hipótesis no es posible investigación alguna, ya que carecería de fundamente científico.

\section{Referencias bibliográficas}

Gimeno, F. 2008. La situación sociolingüística de la Comunidad Valenciana. En Doppelbauer, M. \& Cichon, P. (eds.), La España multilingüe. Lenguas y políticas lingüisticas de España, 213-232. Viena: Praesens.

Gimeno, F. \& Valozic, L. 2012. El impacto de la globalización económica y del anglicismo léxico en los diarios y la publicidad, I Jornadas Vocento. Logroño. http://www.futuroenespañol.es.

Rodrigo, J. 2015. Situación sociolingüística de la lengua de señas española en la Comunidad Valenciana. Mannheim: Publicia. 